\title{
Is Subcutaneous Route an Alternative to Intravenous Route for Mouse Contrast-Enhanced Magnetic Resonance Imaging at $1.5 \mathrm{~T}$ ?
}

\author{
Jean-Philippe Dillenseger, ${ }^{1,2,3}$ Christian Goetz, ${ }^{1,4}$ Amira Sayeh, ${ }^{1,5}$ \\ Pierre-Emmanuel Zorn, ${ }^{1,2,3,6}$ Stéphane Kremer, ${ }^{2,3,6}$ Yves Rémond, ${ }^{2,7}$ André Constantinesco, ${ }^{1}$ \\ Gaëlle Aubertin-Kirch, ${ }^{8}$ and Philippe Choquet $\mathbb{D}^{1,2,3,9}$ \\ ${ }^{1}$ Imagerie Préclinique-UF6237, Hautepierre, Pôle d'Imagerie, Hôpitaux Universitaires de Strasbourg, Strasbourg, France \\ ${ }^{2} I C u b e$ (MMB, AVR, IMIS)-UMR 7357, CNRS, Université de Strasbourg, Strasbourg, France \\ ${ }^{3}$ Fédération de Médecine Translationnelle de Strasbourg, Faculté de Médecine, Université de Strasbourg, Strasbourg, France \\ ${ }^{4}$ Klinik für Nuklearmedizin, Universitätsklinikum, Freiburg, Germany \\ ${ }^{5}$ Service de Radiologie Dentaire-UF8609, Pôle de Médecine et de Chirurgie Bucco-Dentaires, Hôpitaux Universitaires de Strasbourg, \\ Strasbourg, France \\ ${ }^{6}$ Service de Radiologie 2, Hautepierre, Pôle d'Imagerie, Hôpitaux Universitaires de Strasbourg, Strasbourg, France \\ ${ }^{7}$ INSIS-UMR 8622, CNRS, Paris, France \\ ${ }^{8}$ Medical Imaging Analysis Center (MIAC AG), Basel, Switzerland \\ ${ }^{9}$ Laboratory for Adaptable MRI Technology, Department of Biomedical Engineering, University of Basel, Basel, Switzerland
}

Correspondence should be addressed to Philippe Choquet; pchoquet@unistra.fr

Received 24 March 2019; Revised 4 May 2019; Accepted 27 May 2019; Published 13 June 2019

Academic Editor: Yi Wang

Copyright (C) 2019 Jean-Philippe Dillenseger et al. This is an open access article distributed under the Creative Commons Attribution License, which permits unrestricted use, distribution, and reproduction in any medium, provided the original work is properly cited.

\begin{abstract}
The present work compares intravenous (IV) and subcutaneous (SC) routes for contrast-enhanced MRI (CE-MRI) in mice. For that purpose, we selected two contrast media used in clinical practice. MRI acquisitions were performed at $1.5 \mathrm{~T}$ on five adult mice

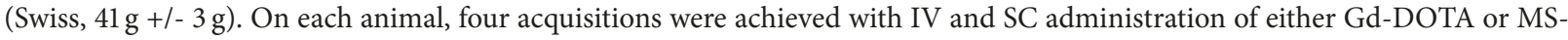
325 ( 1 acquisition per week). For each route, $0.1 \mathrm{~mL}$ of $\mathrm{NaCl}$ and $0.1 \mathrm{~mL}$ of contrast agent were injected. For each acquisition, 200 T1-weighted images were acquired in a $2 \mathrm{~h} 34 \mathrm{~min}$ time lapse. For each route and contrast medium, dynamic contrast enhancement (DCE) curves were obtained. Time-to-peak (TTP), uptake, and washout constant-time values and contrast-to-noise ratio (CNR) were extracted. IV route TTP value was $4.9 \mathrm{~min}$ with Gd-DOTA and $5.4 \mathrm{~min}$ with MS-325. SC route TTP was $43.3 \mathrm{~min}$ with GdDOTA and 45.0 min with MS-325. Despite slower uptake constant-time, we show that SC is a potentially valuable alternative to the IV route in mouse preclinical CE-MRI.
\end{abstract}

\section{Introduction}

Multimodal imaging continues to gain popularity in small animal preclinical research, not only because it satisfies the 3Rs principles (reduction, refinement, and replacement) [1-3], but also because it becomes a fundamental tool of translational medicine. In order to maximize translation from mouse to man, it is highly recommended to share common tomographic imaging procedures like single photon emission computed tomography (SPECT), positron emission tomography (PET), X-Ray Computed Tomography (CT), and nuclear magnetic resonance imaging (MRI) data acquisitions [4-6]. As in clinic, contrast agents for CT and MRI are needed in preclinical imaging in order to enhance tissues, organs, vessels, and lesions, and a considerable amount of laboratory engineering effort has been devoted in recent years to that purpose in order to develop specific MRI contrast agents [7-9]. For all that, standard clinical contrast agents [10] are also widely used in preclinical MRI: they are typically smallmolecular-weight compounds that distribute nonspecifically 
TABLE 1: Acquisition parameters for dynamic CE-MRI imaging.

\begin{tabular}{lc}
\hline Parameters & Values \\
\hline Sequence type & Spoiled gradient recalled echo-2D \\
TR/TE/FA & $25 \mathrm{~ms} / 4 \mathrm{~ms} / 90^{\circ}$ \\
Slice thickness & $1.75 \mathrm{~mm}$ \\
Orientation & $\mathrm{Coronal}$ \\
FOV dimensions (read x phase) & $80 \mathrm{x}^{2} 40 \mathrm{~mm}^{2}$ \\
Reconstructed matrix size & $128 \times 64$ \\
Reconstructed pixel size & $0.625 \times 0.625 \mathrm{~mm}^{2}$ \\
Readout BW & $30 \mathrm{kHz}^{2}$ \\
Nex & 6 \\
Image acquisition time & $4.8 \mathrm{~s}$ \\
Total image number & 200 \\
Acquisition rate for images 1-50 & $12.5 \mathrm{images} \mathrm{per} \mathrm{min}$ \\
Acquisition rate for image 51-200 & 1 image per min \\
Total acquisition duration & $2 \mathrm{~h} \mathrm{34min}$ \\
\hline
\end{tabular}

in the blood plasma and extracellular space of the body after administration [4]. These compounds have the advantage of being low-cost than specific products and their use perfectly meets translational expectations.

One drawback that could critically limit imaging in mice, unlike in humans, is the repeated tail intravenous (IV) puncture, especially when longitudinal procedures are involved. In fact, multiple IV injections result in an increasing risk of tail necrosis around the puncture site [11]. So, it is recommended to carry out the minimum number of needle punctures consistent with good scientific practice. Instead of always using the same puncture site, several different ones should be used along a tail vein [12], which could limit the number of sample times for longitudinal studies. In order to preserve tail veins, subcutaneous (SC) could be an alternative to IV route. To our knowledge, there is no reference describing the use of clinical gadolinium contrast agent for contrast-enhanced MRI (CE-MRI) using the SC route in mice. The aim of this study is to compare IV and SC routes for morphological CE-MRI in mice, with clinical MRI contrast agents with different properties.

\section{Materials and Methods}

\subsection{MRI System and Acquisition Parameters}

2.1.1. MRI System. The MR system (Optimouse 1.5, RS2D, Mundolsheim, France) uses a horizontal magnetic field of 1.5 $\mathrm{T}$ delivered by a cryogen free cryocooled actively shielded superconducting magnet (Cryomagnetics, Oak Ridge, USA). Strength of encoding gradients (Magnex Agilent, Santa Clara, $\mathrm{CA}$, USA) is $20 \mathrm{mT} / \mathrm{m} / \mathrm{A}$ with a maximum reached in $0.1 \mathrm{~ms}$. A $10 \mathrm{~cm}$ long bird-cage RF coil (Rapid Biomedical, Würzburg-Rimpar, Germany) of $40 \mathrm{~mm}$ diameter was used for mouse imaging. MR series in DICOM format are automatically exported to the OsiriX open-source multimodality medical imaging software for further analysis [13].

2.1.2. MRI DCE Acquisition Parameters. For DCE [14-16], dynamic 2D spoiled gradient echo T1-weighted imaging was performed using the keyhole-imaging technique [17, 18]. Acquisition parameters are summarized in Table 1. In order to follow the contrast media enhancement over a sufficient period of time, 200 images were acquired in $2 \mathrm{~h} 34 \mathrm{~min}$, for each animal and each protocol.

2.2. MRI Contrast Agents. The characteristics of the two contrast agents used in this study are summarized in Table 2.

2.2.1. Dotarem (Gd-DOTA). Gd-DOTA (Dotarem, Guerbet, Roissy, France) is a paramagnetic macrocyclic ionic contrast agent developed for MRI [19-23]. It is a sterile, nonpyrogenic, clear, colorless to yellow, aqueous solution of $0.5 \mathrm{mmol} / \mathrm{mL}$ of gadoterate meglumine. Each $\mathrm{mL}$ of Gd-DOTA contains $376.9 \mathrm{mg}$ of gadoterate meglumine, $0.25 \mathrm{mg}$ of DOTA, and water for injection. After intravenous injection, it is confined to the extracellular space and shortens the $\mathrm{T} 1$ and $\mathrm{T} 2$ relaxation times of target tissues. At recommended doses (Table 2), the effect is observed with the greatest sensitivity on T1weighted sequences [24]. Gd-DOTA does not cross the intact blood-brain barrier and, therefore, does not enhance normal brain tissues or lesions that keep a normal blood-brain barrier, e.g., cysts or mature postoperative scars. However, disruption of the blood-brain barrier or abnormal vascularity allows distribution of gadoterate in lesions such as neoplasms, abscesses, or infarcts.

2.2.2. Vasovist (MS-325). MS-325 (Vasovist, Bayer Schering Pharma, Berlin, Germany) is a sterile, nonpyrogenic formulation of a stable gadolinium diethylenetriaminepentaacetic acid chelate derivative with a diphenylcyclohexylphosphate group [19-22, 25]. Each $\mathrm{mL}$ of MS-325 contains $244 \mathrm{mg}$ of gadofosveset trisodium $(0.25 \mathrm{mmol}), 0.27 \mathrm{mg}$ of fosveset, and water for injection. Following IV administration, MS325 binds reversibly to endogenous serum albumin resulting in longer vascular residence time than non-protein binding contrast agents. The binding to serum albumin also increases the magnetic resonance relaxivity and decreases the relaxation time (T1) of water protons in the vicinity 
TABLE 2: Dotarem and Vasovist MRI contrast agent characteristics [10].

\begin{tabular}{|c|c|c|}
\hline Contrast agent (trade name) & Dotarem & Vasovist \\
\hline Molecule name & Gadoterate meglumine & Gadofosveset trisodium \\
\hline Acronym & Gd-DOTA & MS -325 \\
\hline Manufacturer & Guerbet & Bayer Schering \\
\hline Human body region(s) approved & CNS, whole body & Vessels \\
\hline Approved dose $(\mathrm{mmol} / \mathrm{kg})$ & $0.1-0.3$ & 0.03 \\
\hline Concentration & $0.5 \mathrm{~mol} / \mathrm{L}$ & $0.25 \mathrm{~mol} / \mathrm{L}$ \\
\hline Charge & Ionic & Ionic \\
\hline Molecular structure & Cyclic & Linear \\
\hline $\begin{array}{l}\text { Osmolality }\left(\mathrm{mOsm} / \mathrm{kg} \mathrm{H}_{2} \mathrm{O} \text { at }\right. \\
\left.37^{\circ} \mathrm{C}\right)\end{array}$ & 1350 & 825 \\
\hline Viscosity (mPa.s at $\left.37^{\circ} \mathrm{C}\right)$ & 2.0 & 2.1 \\
\hline $\begin{array}{l}\text { Plasmatic relaxivities }\left(r_{1} / r_{2} 1.5 T\right. \\
\left.\text { in L.mmol }{ }^{-1} \cdot \mathrm{s}^{-1}\right)\end{array}$ & $(3.4-3.8) /(3.4-5.2)$ & $(18-20) /(32-36)$ \\
\hline
\end{tabular}

resulting in an increase in signal intensity of blood in T1weighted sequences. In human studies, gadofosveset substantially shortened blood T1 values for up to 4 hours after IV bolus injection [24].

2.3. Animal Management. In order to follow the recommendation to reduce the number of individuals proposed by the 3 Rs ethical rule, MRI acquisitions were performed on five Swiss mice (male, $41 \mathrm{~g} \pm 3 \mathrm{~g}$; 58-62 weeks old). All experimental procedures were approved by the Institutional Animal Care Committee and were done according to French regulations concerning small animal experimentation. On each animal $(n=5), 4$ acquisitions were achieved at one-week intervals (1 acquisition per week per mouse):

(i) IV and SC acquisitions with Gd-DOTA administration;

(ii) IV and SC acquisitions with MS-325 administration. For all acquisitions $(n=20)$, mice were kept anesthetized with isoflurane (1-1.5\% in air), were monitored (respiration), and warmed at mouse body temperature in imaging cell (Minerve, Esternay, France) positioned at the middle of the MRI coil.

2.4. DCE Protocol. For the IV route acquisitions, a needle was placed in a tail vein. For the SC route acquisitions, a catheter was placed at the mediodorsal part of the pelvis. For each route, the injection point was connected to an automatic syringe pump (Harvard Apparatus, Holliston, USA) via catheter tubing. Its length corresponds to a volume of $0.1 \mathrm{~mL}$. The catheter tubing was filled with $0.1 \mathrm{~mL} \mathrm{NaCl}$ serum and connected to a syringe containing the contrast agent (Figure 1). For each route, $0.1 \mathrm{~mL} \mathrm{NaCl}$ (catheter tubing) followed by $0.1 \mathrm{~mL}$ of contrast agent (syringe) was injected in 2 minutes. The DCE MRI sequence and injection started simultaneously.

2.5. Data Processing. For each route (IV and SC), the DCE data [14] were extracted with the OsiriX region of interest (ROI) enhancement plugin tool [13]. ROIs were drawn

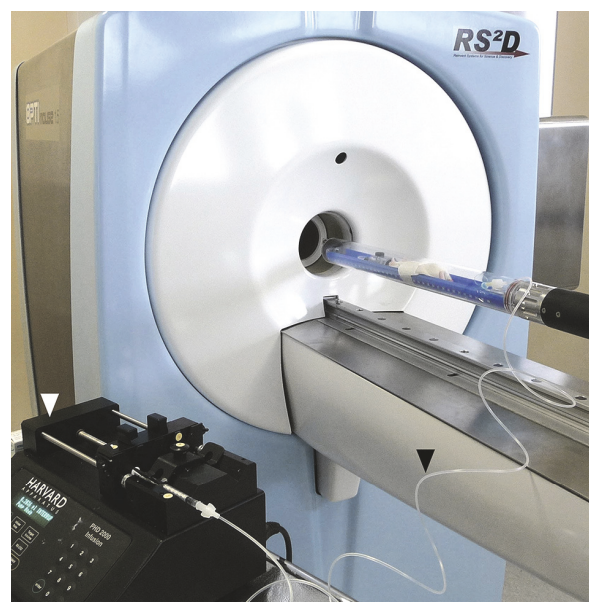

FIGURE 1: Injection system (white arrow head) positioned nearby the $1.5 \mathrm{~T}$ preclinical MRI connected to the mouse via intravenous or subcutaneous catheter (black arrow head).

around structures of interest (liver, lower vena cava, and muscles) (Figure 2). Mean DCE curves ( $n=5$ mice) were obtained for each injection route and curve fitting (ProFit, QuantumSoft, Uetikon am See, Switzerland) was done for each organ of interest.

Mean organ uptake and washout constant times were obtained from the fitting curve $\mathrm{f}(\mathrm{t})(1)$, derived from a classical monocompartmental model using a bolus extra vascular input function, where $T_{U}$ is the tissue uptake constant time and $\mathrm{T}_{\mathrm{W}}$ is the corresponding washout constant time. $\mathrm{K}$ is a constant depending on both $\mathrm{Tu}$ and $\mathrm{Tw}$ and the fraction of the administered dose.

$$
f(t)=K \cdot\left[e^{\left(-t / T_{w}\right)}-e^{\left(-t / T_{U}\right)}\right]
$$

For each organ of interest $i$ (e.g., liver, lower vena cava, and muscles), the mean contrast to noise ratio $\left(\mathrm{mCNR}_{i}\right)$ was also measured ( $n=5$ mice), with OsiriX [13], between the first acquisition $(t=T 0)$ and the maximum enhanced image set 

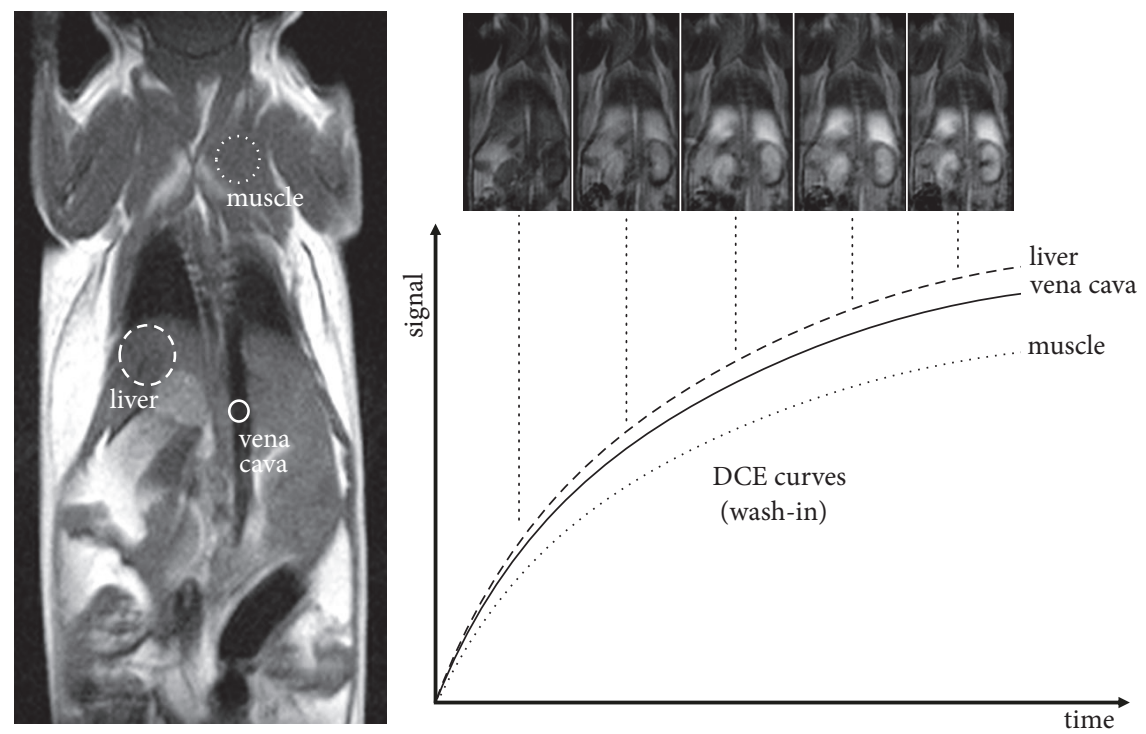

FIGURE 2: Illustration of liver, muscle, and vena cava ROI positions to generate MRI DCE curves (only wash-in part is schematically represented in this illustration).

$(\mathrm{t}=$ time to peak (TTP)), using the mean ROI value (mROI) and the mean standard deviation (mSD), according to the following formula:

$$
\mathrm{mCNR}_{i}=\frac{\left|\mathrm{mROI}_{\mathrm{TTP}}-\mathrm{mROI}_{\mathrm{T} 0}\right|}{\sqrt{\mathrm{mSD}_{\mathrm{TTP}}^{2}+\mathrm{mSD}_{\mathrm{T} 0}^{2}}}
$$

\section{Results}

All mice $(n=5)$ survived the experiments. During all MRI acquisitions, mice mean respiratory rate was $110 \pm 17$ breaths per minutes.

TTP, uptake, and washout constant times and mCNR results are summarized in Table 3. Figures 3 and 4, respectively, show IV and SC mean enhancement data $( \pm S D)(n=$ 5 mice) and fitting curves from the related regions (liver, muscle, and lower vena cava).

3.1. Uptake Constant Time and TTP Results. All mean values $\pm \mathrm{SD}$ were obtained on 5 mice. IV route mean uptake constant times were the same for Gd-DOTA and for MS325: $1.1 \mathrm{~min}$. The SC route mean uptake constant time was 24.4 min with Gd-DOTA and 21.3 min with MS-325. IV route mean TTP was 4.9 min with Gd-DOTA and 5.4 min with MS325. The SC route mean TTP was $43.3 \mathrm{~min}$ with Gd-DOTA and $45.0 \mathrm{~min}$ with MS-325.

3.2. Washout Constant Time Analysis. All mean values \pm SD were obtained on 5 mice. IV route mean washout constant time was 71.9 min with Gd-DOTA and $143.1 \mathrm{~min}$ with MS325. SC route mean washout constant time was $79.4 \mathrm{~min}$ with Gd-DOTA and $120.5 \mathrm{~min}$ with MS-325. Results verify that MS-325 is a specific contrast agent with a longer vascular residence time [19-22, 25].
3.3. $m$ CNR Results. mCNR values were, for each ROI, lower with Gd-DOTA compared to MS-325 (Table 3). No major mCNR differences were observed between IV and SC routes. Figure 5 presents images at T0 and TTP following IV/SC administration of Gd-DOTA/MS-325.

Figure 6 (and S1 and S2) shows head and neck MR angiographic images obtained with MS-325 via SC and IV administration routes, respectively. The DCE MRI scan delay was adapted for each route, in order to reach the maximum contrast enhancement at mid-acquisition. mCNR values between muscle and right jugular vein were 5.1 in SC route (Figure 6(a)) versus 6.2 in IV route (Figure 6(b)).

\section{Discussion}

4.1. IV Tail Puncture in Mice. Nowadays, the IV route is by far and away the most frequently used in humans for contrast agent administration in most current procedures. IV route is also used in laboratory animals [12]; however, repeated tail vein punctures present some limits.

(i) Repeatability. In mice, IV tail puncture is poorly reproducible over time due to tail necrosis around the puncture sites [11]. This fact limits longitudinal follow-up studies with imaging and even more, multimodal protocols which require deliveries of both tracers and contrast agents for the acquisition of both functional and morphological data. In fact, in human beings, similar difficulties could occur, as repeated vein punctures impose frequent changes of puncture localisations (e.g., hand dorsal veins, upper limb basilic or cephalic vein) and so could require the surgical creation of an arteriovenous fistula, for instance, in the case of haemodialysis [26].

(ii) Technical Limits. With small diameter and fragile vessels, vein puncture could be complex, requires experienced practitioners, and, hence, is operator dependent. 
TABLE 3: TTP and semiquantitative uptake and washout values extracted from DCE analysis.

\begin{tabular}{|c|c|c|c|c|c|c|c|}
\hline \multirow{2}{*}{ Contrast agent } & \multirow{2}{*}{ Fit-curves analysis } & \multicolumn{3}{|c|}{ Intravenous } & \multicolumn{3}{|c|}{ Subcutaneous } \\
\hline & & Liver & Muscle & Vein & Liver & Muscle & Vein \\
\hline \multirow{7}{*}{ Gd-DOTA (Dotarem) } & Uptake constant time (min) & 1.1 & 1.1 & 1.1 & 37.0 & 26.3 & 17.5 \\
\hline & $\begin{array}{c}\text { Mean uptake } \\
\text { (min) }\end{array}$ & & 1.1 & & & 24.4 & \\
\hline & Washout constant time (min) & 80.0 & 58.5 & 81.3 & 75.8 & 61.0 & 120.4 \\
\hline & Mean washout (min) & & 71.9 & & & 79.4 & \\
\hline & $\begin{array}{l}\text { Time to peak (TTP) } \\
\text { (min) }\end{array}$ & 4.9 & 4.8 & 5.0 & 51.1 & 39.0 & 40.0 \\
\hline & $\begin{array}{c}\text { mean time to peak (mTTP) } \\
\text { (min) }\end{array}$ & & 4.9 & & & 43.3 & \\
\hline & $m C N R($ at $m T T P)$ & 7.5 & 2.5 & 3.5 & 5.8 & 1.9 & 2.7 \\
\hline \multirow{7}{*}{ MS-325 (Vasovist) } & Uptake constant time (min) & 1.1 & 1.1 & 1.1 & 19.6 & 24.4 & 20.4 \\
\hline & $\begin{array}{c}\text { Mean uptake } \\
\text { ( } \mathrm{min})\end{array}$ & & 1.1 & & & 21.3 & \\
\hline & Washout constant time (min) & 144.9 & 114.9 & 169.5 & 140.9 & 106.4 & 126.6 \\
\hline & Mean washout (min) & & 143.1 & & & 120.5 & \\
\hline & $\begin{array}{c}\text { Time to peak (TTP) } \\
\text { (min) }\end{array}$ & 5.9 & 5.3 & 5.1 & 44 & 47 & 44 \\
\hline & $\begin{array}{c}\text { Mean time to peak (mTTP) } \\
\text { (min) }\end{array}$ & & 5.4 & & & 45.0 & \\
\hline & $m C N R($ at $m T T P)$ & 10.5 & 9.4 & 20.1 & 10.6 & 13.6 & 18.9 \\
\hline
\end{tabular}
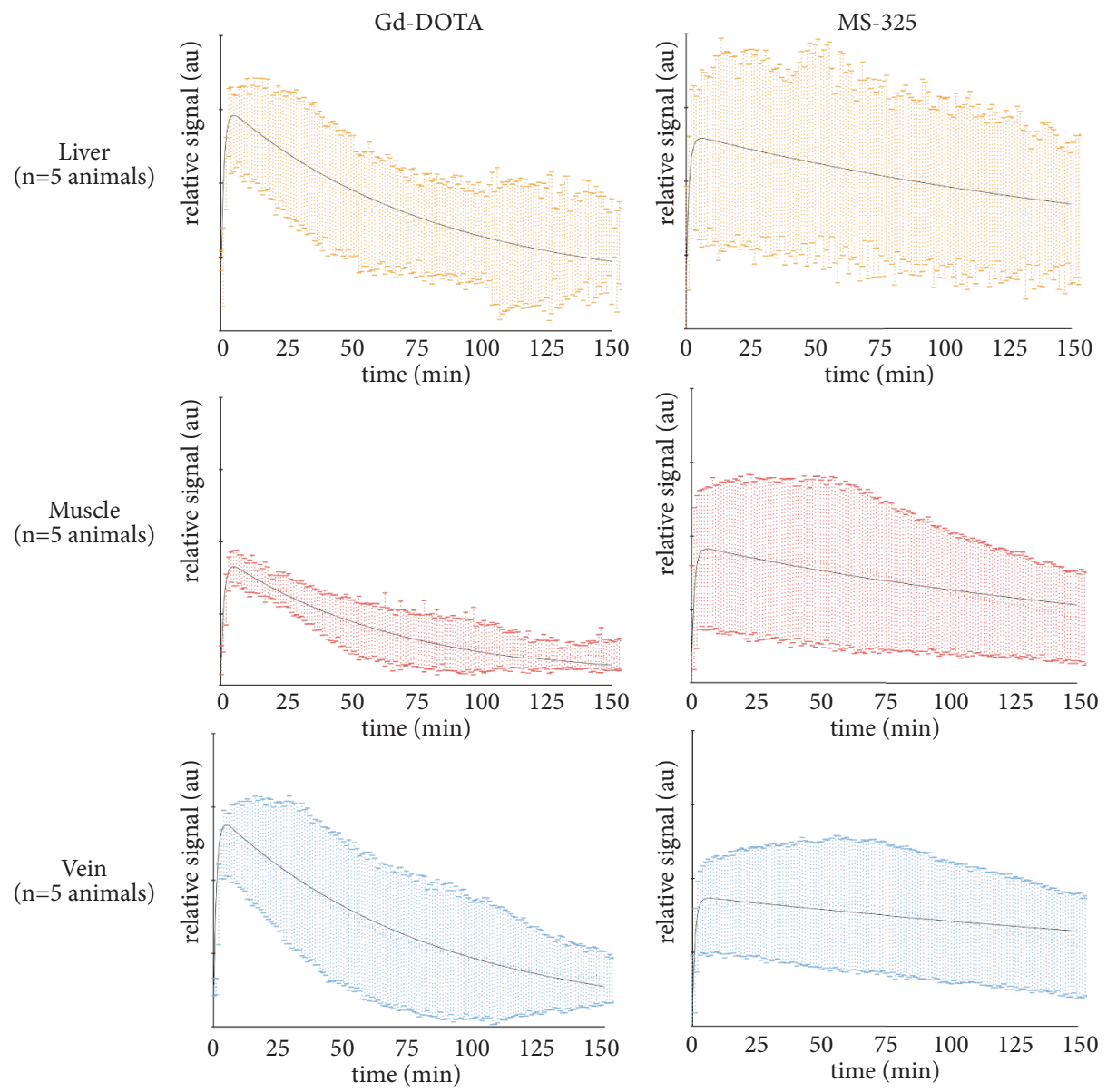

FIGURE 3: DCE data \pm SD (in colors) and fitting curves (black continuous lines) of liver, muscle, and lower vena cava enhancement after IV injection of $0.1 \mathrm{~mL}$ of Gd-DOTA (Dotarem) and MS-325 (Vasovist). 
Subcutaneous route
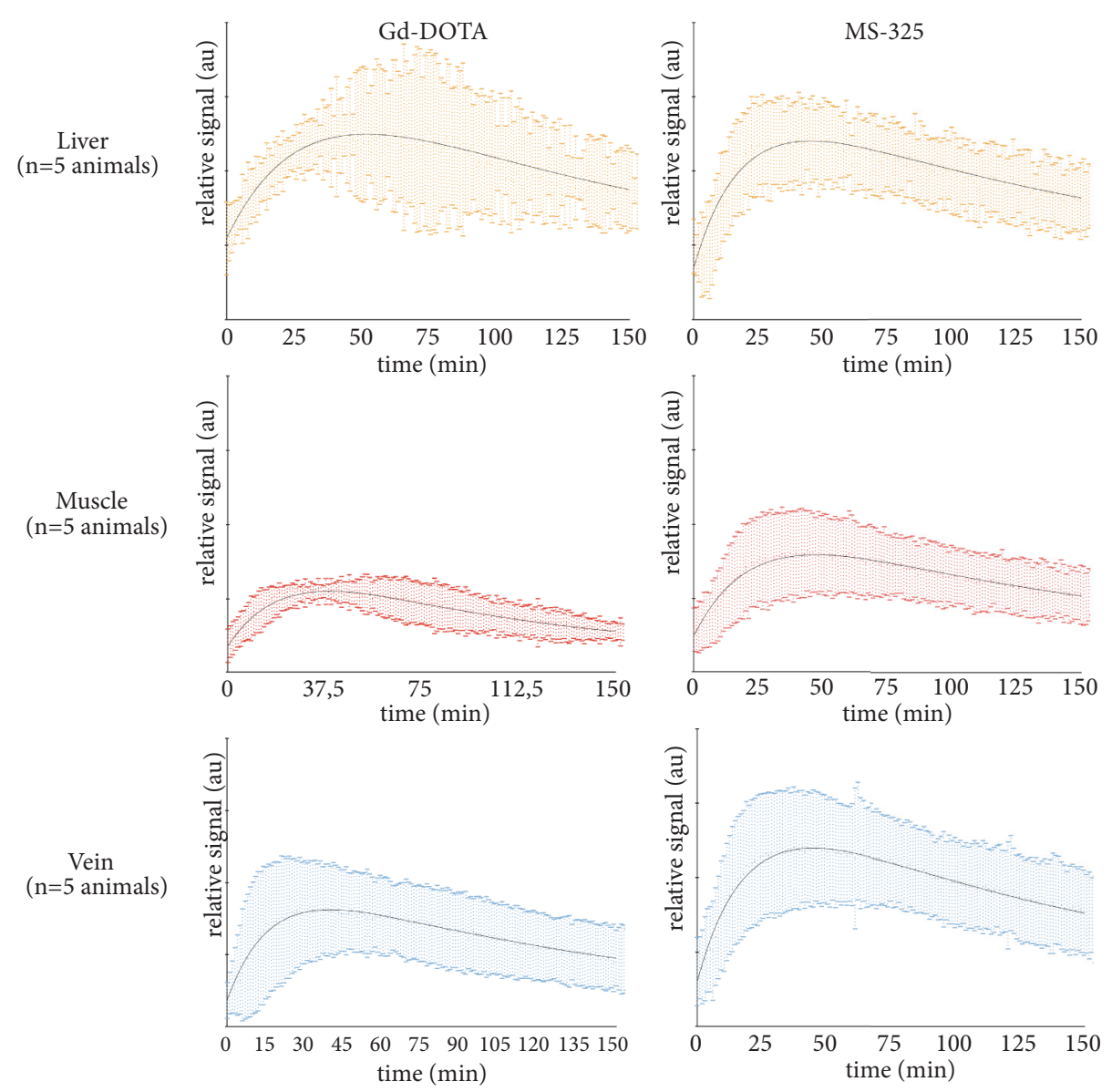

FIGURE 4: DCE data \pm SD (in colors) and fitting curves (black continuous lines) of liver, muscle and lower vena cava enhancement after SC injection of $0.1 \mathrm{~mL}$ of Gd-DOTA (Dotarem) and MS-325 (Vasovist).

A dedicated appropriate workspace is needed to achieve successful punctures in proper conditions [12]. Some research papers tested several small injection ports [27] and presented the developments of automated puncture systems based on vision and/or pressure feedback [11,28], which could be an interesting alternative to manual puncture.

(iii) Physiological Limits. Conventionally, injected contrast media volume is limited by the animal/patient weight. In mice, IV volumes considered good practice are up to $5 \mathrm{ml} . \mathrm{kg}^{-1}[12,29]$. In the preclinical field, it is difficult to optimize injected volumes, especially in the case of multimodal protocols involving the use of different contrast agents or tracers. Avoiding the IV route, at least for one compound, is an advantage by limiting the role amount injected liquid.

(iv) Reproducibility. In the preclinical field, technical and physiological limits could have an impact on reproducibility $[30,31]$. For instance, in practice, it is difficult to ensure that the entire volume is delivered in the vascular compartment only, due to the possibility of extravasation.

All these constraints induce limitations or introduce drawbacks in multimodal imaging and/or longitudinal follow-up. Nowadays, multimodal preclinical platforms with
MRI and SPECT or PET are expanding rapidly. They are designed with separated apparatus [32, 33] or combined apparatus $[4,5]$. In this context, in order to save the use of the tail veins exclusively for radioactive tracer administration for SPECT or PET imaging, the question we seek to answer is whether the SC route could be an alternative to an IV injection during multimodal proceedings involving T1w MRI acquisitions.

4.2. The SC Route: An Overview. The chemical, physical, and physiological properties of the SC injection route are complex and unclear [34]. The fact remains is that the SC route is used as an interesting way for drug delivery in human beings in many fields (e.g., IgC therapy, insulin-dependent therapy, palliative care, and anemia treatment) [35-38]. Nevertheless, in clinical medical imaging, except for lymphoscintigraphy [39], it is poorly described in the relevant literature. In contrary, in small animal imaging, we found a large number of studies that used SC delivery for manganese-based contrast agent especially for mouse or rat brain imaging [40-46] but also for implanted tumours [43, 47]. SC deliveries were also described for iron-based particles for lymph node or tumour 


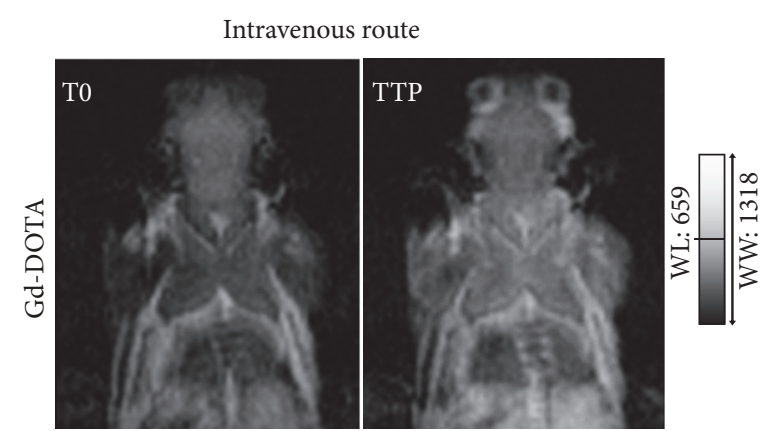

(a)

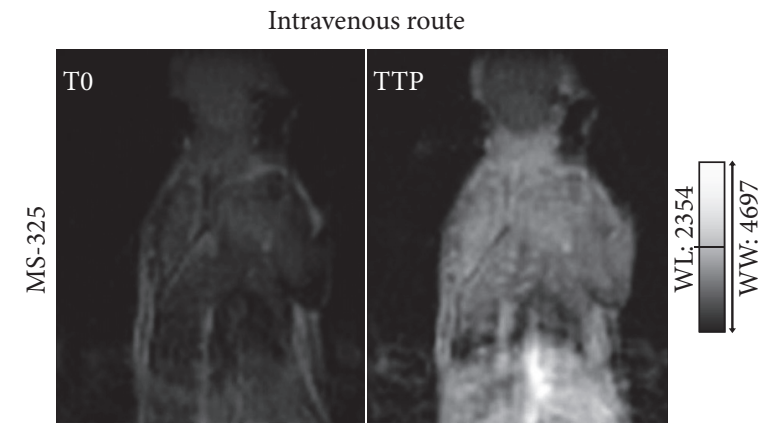

(c)

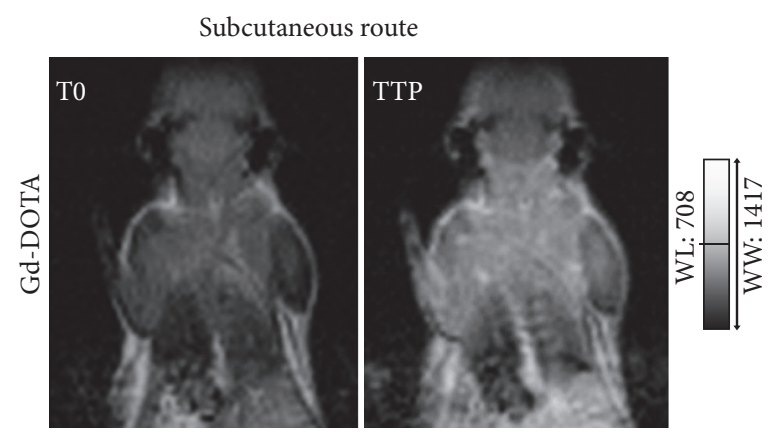

(b)

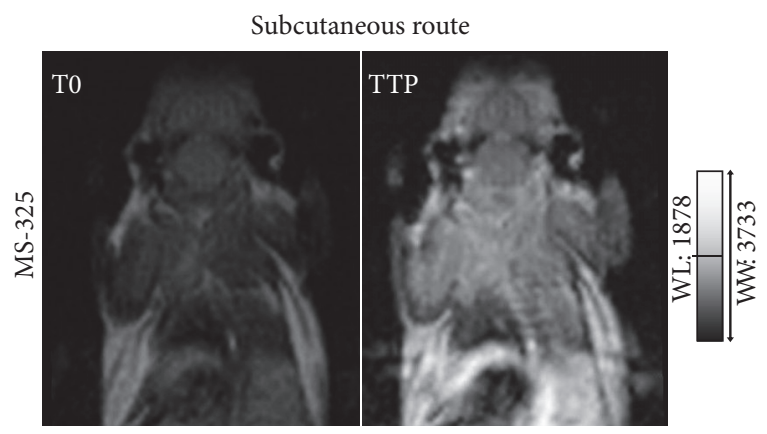

(d)

FiguRE 5: MR images obtained at T0 and TTP. Gd-DOTA IV (a) and SC (b) routes; MS-325 IV (c) and SC (d) routes. Windowing was done on the TTP image full dynamic scale and applied to the T0 image.

imaging $[48,49]$. Dual-doped nanorods or nanospheres for imaging and drug delivery also used SC delivery $[50,51]$ as well as surface modified or multivesicular liposomes containing Gd-DTPA and drugs [52, 53]. Gadolinium liposomal contrast agents were also developed for lymph node MRI [54]. All the previously mentioned contrast media were developed for preclinical research more to target fundamental biological processes than to be used in humans. No studies have yet described the SC use of clinical gadolinium contrast agent for small animal imaging. However, this type of administration presents several advantages in preclinical research:

(i) First, using clinical gadolinium-based agents in preclinical imaging could facilitate result transfers from mouse to man.

(ii) Second, a SC puncture is easy to do technically and, because the SC space is a virtual space, it can also be an excellent site for larger volume fluid delivery: up to $10 \mathrm{~mL} \cdot \mathrm{kg}^{-1}[12,29]$.

4.3. Result Analysis and Practical Consequences. We demonstrate with dynamic CE-MRI curves that SC administration is a potential alternative to the IV route in preclinical imaging. Injection route directly impacts mean uptake constant times and as a consequence TTP values. Mean TTP values were in the range between 4,8 and 5,9 $\mathrm{min}$ in IV route and between 39 and $47 \mathrm{~min}$ in SC route. Concerning $\mathrm{mCNR}_{\mathrm{T} 0 \text {-TTP }}$, the MS-325 contrast agent gives higher values than Gd-DOTA for each route (Table 3 ). This is explained by the higher plasmatic longitudinal relaxivity of MS-325 (Table 2). mCNR results are similar for each route with MS-325, which can be partly accounted for by the fact that MS-325 has a longer vascular residence time $[20,21,25]$. For Gd-DOTA, the SC route quantitatively presents lower $\mathrm{mCNR}_{\mathrm{TO} \text {-TTP }}$ values ($23 \%$ ) than the IV route (Table 3), due to the shorter vascular residence time of this contrast agent [19-23]. In any case, at TTP, contrast enhancement with Gd-DOTA can clearly be seen for both routes (Figure 5).

One observation is still pending: in IV route, the maximum venous enhancement (at TTP) is relatively higher with Gd-DOTA than with MS-325 (Figure 3), whereas the latter has a higher plasmatic relaxivity (Table 2). Our work consists in comparing two different routes (IV and SC): this observation does not affect its main conclusions.

Knowing the SC, TTP mean value may be interesting in daily practice, in helping for contrast enhanced MRI while preserving the animal's tail. TTP value allows time between administration and acquisition. The IV route can thus be saved for compounds that need to be administered via this route (e.g., radiotracers). By keeping the SC route for MRI and the IV route for SPECT or PET, it is easy to complete both kinds of explorations in the same time lapse. In daily practice, the $\mathrm{SC}$ route allows to save the time initially required for the placement of a venous catheter (if no other injection is needed). From a technical point of view, a SC puncture is easier than an IV puncture; as a consequence SC is potentially more repeatable and reproducible. 

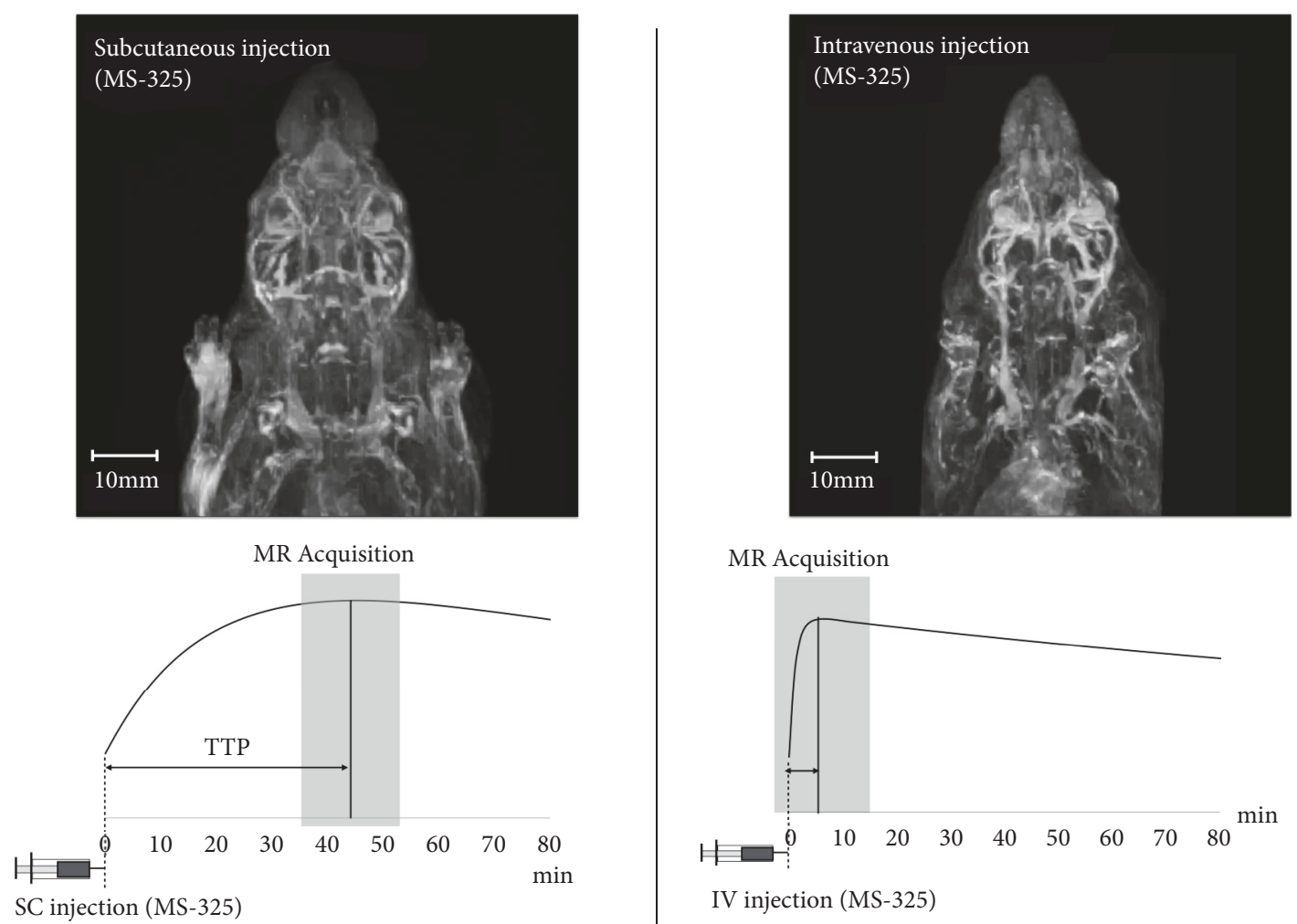

IV injection (MS-325)

(a)

(b)

\begin{tabular}{|c|c|c|c|}
\hline MRI system & Optimouse $1.5 \mathrm{~T}$ & FOV dimensions & $80 \times 40 \mathrm{~mm}$ \\
\hline Sequence type & $\begin{array}{l}\text { Spoiled gradient recalled echo- } \\
\text { 3D }\end{array}$ & $\begin{array}{l}\text { Reconstructed Matrix } \\
\text { size }\end{array}$ & $256 \times 126$ \\
\hline Sequence name & SP-GRE 3D & Pixel size & $312.5 \times 312.5 \mu \mathrm{m}^{2}$ \\
\hline TR/TE/FA & $30 \mathrm{~ms} / 6 \mathrm{~ms} / 40^{\circ}$ & Readout BW & $30 \mathrm{kHz}$ \\
\hline Slice thickness & $312.5 \mu \mathrm{m}$ & Nex & 2 \\
\hline Orientation & Coronal & Total acquisition duration & 16 minutes \\
\hline
\end{tabular}

FIGURE 6: MIP view of the head of a mouse following SC (a) and IV (b) injection of MS-325 (Vasovist), and curves showing the delay from administration and duration of MR acquisition (see S1 and S2). Acquisition parameters were presented in (c).

4.4. Considerations on the Intraperitoneal (IP) Route. The IP route is another administration route, widely used in small animals [12] which could also be an alternative for repeated IV tail punctures. From a technical viewpoint, this administration mode is simpler than IV puncture but more challenging than the SC route.

In fact, although the IP route is considered a routine route of administration in rodents, it has several limitations and potential risks $[55,56]$. These include pain, potential peritoneum irritation, formation of adhesions within the abdominal cavity, and needle perforation (organs, bowels, and abdominal vessels) $[15,55]$. In addition, repeated administration may result in cumulative irritant effects and needleinduced damage [29]. Furthermore, IP administration might result in injection into muscle, subcutaneous tissue, and the intestine and other organs rather than injection exclusively into the peritoneal cavity. Underestimation of the number of failures is also suspected [55].

Also, the presence of contrast agent in the peritoneal cavity may, if the study concerns the abdominal-pelvic region, interfere with the analysis of contrast enhancement of vascular or pathological structures (e.g., mice models with intra-abdominal tumours).

Furthermore, careful aseptic conditions are needed, as well are operators that are well trained in the handling and restraint of laboratory animals [57].

Although IP delivery is a parenteral route of administration, the pharmacokinetics of substances administered intraperitoneally are more akin to those observed after oral administration: the primary route of absorption is via the mesenteric vessels, which drain into the portal vein and pass through the liver $[12,58]$. 


\section{Conclusion}

The SC route could be a valuable alternative to the IV route in small-animal CE MRI. This contrast administration route allows, on the one hand, preserving the tail veins of the animal and, on the other, saving the time needed for the placement of a venous catheter. Subcutaneously obtained CE can be as efficient as intravenously obtained enhancement for morphological MRI applications. The SC route can definitely be regarded as a useful choice for preclinical longitudinal studies involving MRI with other modalities that require the use of an IV route (e.g., radiotracer administration in PET or SPECT). The potential of this administration route remains to be evaluated with other MR contrast agents.

\section{Data Availability}

The data used to support the findings of this study are available from the corresponding author (pchoquet@unistra.fr) upon motivated request.

\section{Conflicts of Interest}

S. Kremer is a member of the French national medical board of Bayer Schering Pharma, without remuneration. The other authors of this manuscript declare no relationships with any companies, whose products or services may be related to the subject matter of the article.

\section{Acknowledgments}

The authors warmly thank Nadine Mischler for manuscript editing.

\section{Supplementary Materials}

Supplementary 1. S1. MIP reconstruction from head and neck mouse MR acquisition after subcutaneous (SC) injection of Vasovist. (MPEG 4.7 Mo).

Supplementary 2. S2. MIP reconstruction from head and neck mouse MR acquisition after intravenous (IV) injection of Vasovist. (MPEG 4.2 Mo).

\section{References}

[1] Y. Ohno, "ICH guidelines - Implementation of the 3Rs (refinement, reduction, and replacement): Incorporating best scientific practices into the regulatory process," ILAR Journal, vol. 43, pp. S95-S98, 2002.

[2] B. Rusche, "The 3 Rs and animal welfare - conflict or the way forward?” ALTEX, vol. 20, pp. 63-76, 2003.

[3] F. Guhad, "Introduction to the 3Rs (refinement, reduction and replacement)," Journal of the American Association for Laboratory Animal Science, vol. 44, pp. 58-59, 2005.

[4] R. A. Frank, B. Långström, G. Antoni et al., "The imaging continuum: bench to biomarkers to diagnostics," Journal of Labelled Compounds and Radiopharmaceuticals, vol. 50, no. 910, pp. 746-769, 2007.
[5] B. J. Pichler, M. S. Judenhofer, and C. Pfannenberg, "Multimodal imaging approaches: PET/CT and PET/MRI," in Molecular Imaging I, vol. 185/1 of Handbook of Experimental Pharmacology, pp. 109-132, Springer, Berlin, Germany, 2008.

[6] H. Cho, E. Ackurstaff, S. Carlin et al., "Noninvasive multimodality imaging of the tumor microenvironment: registered dynamic magnetic resonance imaging and positron emission tomography studies of a preclinical tumor model of tumor hypoxia," Neoplasia, vol. 11, no. 3, pp. 247-259, 2009.

[7] H. Kobayashi, S. Kawamoto, R. A. Star, T. A. Waldmann, Y. Tagaya, and M. W. Brechbiel, "Micro-magnetic resonance lymphangiography in mice using a novel dendrimer-based magnetic resonance imaging contrast agent," Cancer Research, vol. 63, no. 2, pp. 271-276, 2003.

[8] K. B. Ghaghada, M. Ravoori, D. Sabapathy et al., "New dual mode gadolinium nanoparticle contrast agent for magnetic resonance imaging," PLoS ONE, vol. 4, no. 10, p. e7628, 2009.

[9] M. R. Bashir, L. Bhatti, D. Marin, and R. C. Nelson, "Emerging applications for ferumoxytol as a contrast agent in MRI," Journal of Magnetic Resonance Imaging, vol. 41, pp. 884-898, 2015.

[10] D. Hao, T. Ai, F. Goerner, X. Hu, V. M. Runge, and M. Tweedle, "MRI contrast agents: Basic chemistry and safety," Journal of Magnetic Resonance Imaging, vol. 36, no. 5, pp. 1060-1071, 2012.

[11] Y.-C. Chang, B. Berry-Pusey, R. Yasin et al., "An automated mouse tail vascular access system by vision and pressure feedback," IEEE/ASME Transactions on Mechatronics, vol. 20, no. 4, pp. 1616-1623, 2015.

[12] K.-H. Diehl, R. Hull, D. Morton et al., "A good practice guide to the administration of substances and removal of blood, including routes and volumes," Journal of Applied Toxicology, vol. 21, no. 1, pp. 15-23, 2001.

[13] A. Rosset, L. Spadola, and O. Ratib, "OsiriX: an open-source software for navigating in multidimensional DICOM images," Journal of Digital Imaging, vol. 17, no. 3, pp. 205-216, 2004.

[14] S. L. Barnes, J. G. Whisenant, M. E. Loveless, and T. E. Yankeelov, "Practical dynamic contrast enhanced mri in small animal models of cancer: data acquisition, data analysis, and interpretation," Pharmaceutics, vol. 4, no. 3, pp. 442-478, 2012.

[15] T. E. Yankeelov and J. C. Gore, "Dynamic contrast enhanced magnetic resonance imaging in oncology: theory, data acquisition, analysis, and examples," Current Medical Imaging Reviews, vol. 3, no. 2, pp. 91-107, 2007.

[16] K. Hittmair, G. Gomiscek, K. Langenberger, M. Recht, H. Imhof, and J. Kramer, "Method for the quantitative assessment of contrast agent uptake in dynamic contrast-enhanced MRI," Magnetic Resonance in Medicine, vol. 31, no. 5, pp. 567-571, 1994.

[17] J. J. Van Vaals, M. E. Brummer, W. Thomas Dixon et al., "“Keyhole" method for accelerating imaging of contrast agent uptake," Journal of Magnetic Resonance Imaging, vol. 3, no. 4, pp. 671-675, 1993.

[18] J. E. Bishop, G. E. Santyr, F. Kelcz, and D. B. Plewes, "Limitations of the keyhole technique for quantitative dynamic contrastenhanced breast MRI," Journal of Magnetic Resonance Imaging, vol. 7, no. 4, pp. 716-723, 1997.

[19] A. Chachuat, P. Molinier, B. Bonnemain, C. Chambon, and J. L. Gayet, "Pharmacokinetics and tolerance of Gd-DOTA (DOTAREM) in healthy volunteers and in patients with chronic renal failure," European Radiology, vol. 2, no. 4, pp. 326-329, 1992.

[20] H. Shokrollahi, "Contrast agents for MRI," Materials Science and Engineering C: Materials for Biological Applications, vol. 33, no. 8, pp. 4485-4497, 2013. 
[21] E. A. Waters and S. A. Wickline, "Contrast agents for MRI," Basic Research in Cardiology, vol. 103, no. 2, pp. 114-121, 2008.

[22] M.-F. Bellin and A. J. Van Der Molen, "Extracellular gadolinium-based contrast media: an overview," European Journal of Radiology, vol. 66, no. 2, pp. 160-167, 2008.

[23] P. Caravan, J. J. Ellison, T. J. McMurry, and R. B. Lauffer, "Gadolinium(III) chelates as MRI contrast agents: structure, dynamics, and applications," Chemical Reviews, vol. 99, no. 9, pp. 2293-2352, 1999.

[24] Y. Shen, F. L. Goerner, C. Snyder et al., "T1 relaxivities of gadolinium-based magnetic resonance contrast agents in human whole blood at 1.5, 3, and 7 T,' Investigative Radiology, vol. 50, no. 5, pp. 330-338, 2015.

[25] T. Steger-Hartmann, P. B. Graham, S. Müller, and H. Schweinfurth, "Preclinical safety assessment of Vasovist (Gadofosveset trisodium), a new magnetic resonance imaging contrast agent for angiography," Investigative Radiology, vol. 41, no. 5, pp. 449459, 2006.

[26] S. B. Leapman, M. Boyle, M. D. Pescovitz et al., "The arteriovenous fistula for hemodialysis access: gold standard or archaic relic?" The American Journal of Surgery, vol. 62, pp. 652-656, 1996.

[27] T. Fiebig, G. Figueiredo, H. Boll et al., "A low cost metal-free vascular access mini-port for artifact free imaging and repeated injections in mice," PLoS ONE, vol. 8, no. 6, p. e65939, 2013.

[28] B. N. Berry-Pusey, Y. C. Chang, S. W. Prince et al., "A semiautomated vascular access system for preclinical models," Physics in Medicine and Biology, vol. 58, no. 16, pp. 5351-5362, 2013.

[29] P. V. Turner, T. Brabb, C. Pekow, and M. A. Vasbinder, "Administration of substances to laboratory animals: routes of administration and factors to consider," Journal of the American Association for Laboratory Animal Science, vol. 50, pp. 600-613, 2011.

[30] D. C. Vines, D. E. Green, G. Kudo, and H. Keller, "Evaluation of mouse tail-vein injections both qualitatively and quantitatively on small-animal PET tail scans," Journal of Nuclear Medicine Technology, vol. 39, no. 4, pp. 264-270, 2011.

[31] C. Lasnon, A. E. Dugué, M. Briand, S. Dutoit, and N. Aide, "Quantifying and correcting for tail vein extravasation in small animal PET scans in cancer research: is there an impact on therapy assessment?" EJNMMI Research, vol. 5, no. 1, pp. 61-65, 2015.

[32] J. Dillenseger, B. Guillaud, C. Goetz et al., "Coregistration of datasets from a micro-SPECT/CT and a preclinical $1.5 \mathrm{~T}$ MRI," Nuclear Instruments and Methods in Physics Research Section A: Accelerators, Spectrometers, Detectors and Associated Equipment, vol. 702, pp. 144-147, 2013.

[33] C. Goetz, E. Breton, P. Choquet, V. Israel-Jost, and A. Constantinesco, "SPECT low-field MRI system for small-animal imaging," Journal of Nuclear Medicine, vol. 49, no. 1, pp. 88-93, 2008.

[34] H. M. Kinnunen and R. J. Mrsny, "Improving the outcomes of biopharmaceutical delivery via the subcutaneous route by understanding the chemical, physical and physiological properties of the subcutaneous injection site," Journal of Controlled Release, vol. 182, no. 1, pp. 22-32, 2014.

[35] R. Bellazzi, G. Nucci, and C. Cobelli, "The subcutaneous route to insulin dependent diabetes therapy," IEEE Engineering in Medicine and Biology Magazine, vol. 20, no. 1, pp. 54-64, 2001.
[36] M. Berger, "Subcutaneous administration of IgG," Immunology and Allergy Clinics of North America, vol. 28, no. 4, pp. 779-802, 2008.

[37] C. Fonzo-Christe, C. Vukasovic, A. Wasilewski-Rasca, and P. Bonnabry, "Subcutaneous administration of drugs in the elderly: survey of practice and systematic literature review," Palliative Medicine, vol. 19, no. 3, pp. 208-219, 2005.

[38] T. V. Patel, K. Robinson, and A. K. Singh, "Is it time to reconsider subcutaneous administration of epoetin?" Nephrology News \& Issues, vol. 21, pp. 57-59, 2007.

[39] S. M. Moghimi and B. Bonnemain, "Subcutaneous and intravenous delivery of diagnostic agents to the lymphatic system: applications in lymphoscintigraphy and indirect lymphography," Advanced Drug Delivery Reviews, vol. 37, no. 1-3, pp. 295312, 1999.

[40] M. S. Shazeeb and C. H. Sotak, "Dose dependence and temporal evolution of the T1 relaxation time and MRI contrast in the rat brain after subcutaneous injection of manganese chloride," Magnetic Resonance in Medicine, vol. 68, no. 6, pp. 1955-1962, 2012.

[41] Y. Kuo, A. H. Herlihy, P. So, K. K. Bhakoo, and J. D. Bell, "In vivo measurements of $\mathrm{T} 1$ relaxation times in mouse brain associated with different modes of systemic administration of manganese chloride," Journal of Magnetic Resonance Imaging, vol. 21, no. 4, pp. 334-339, 2005.

[42] T. Watanabe, O. Natt, S. Boretius, J. Frahm, and T. Michaelis, "In vivo 3D MRI staining of mouse brain after subcutaneous application of $\mathrm{MnCl}_{2}$," Magnetic Resonance in Medicine, vol. 48, no. 5, pp. 852-859, 2002.

[43] O. Eschenko, S. Canals, I. Simanova, M. Beyerlein, Y. Murayama, and N. K. Logothetis, "Mapping of functional brain activity in freely behaving rats during voluntary running using manganese-enhanced MRI: implication for longitudinal studies," NeuroImage, vol. 49, no. 3, pp. 2544-2555, 2010.

[44] M. R. Sepúlveda, T. Dresselaers, P. Vangheluwe et al., "Evaluation of manganese uptake and toxicity in mouse brain during continuous $\mathrm{MnCl} 2$ administration using osmotic pumps," Contrast Media \& Molecular Imaging, vol. 7, no. 4, pp. 426-434, 2012.

[45] A. C. Silva and N. A. Bock, "Manganese-enhanced MRI: an exceptional tool in translational neuroimaging," Schizophrenia Bulletin, vol. 34, no. 4, pp. 595-604, 2008.

[46] F. Chen, Z. Gu, H. Wan, X. Xu, and Q. Tang, "Manganese nanosystem for new generation of MRI contrast agent," Reviews in Nanoscience and Nanotechnology, vol. 4, no. 2, pp. 81-91, 2015.

[47] T. Ganesh, R. B. Mokhtari, M. Alhamami et al., "Manganese-enhanced MRI of minimally gadolinium-enhancing breast 459 tumors," Journal of Magnetic Resonance Imaging, vol. 41, pp. 806-813, 2015.

[48] Y. Mori, M. Umeda, M. Fukunaga, K. Ogasawara, and Y. Yoshioka, "MR contrast in mouse lymph nodes with subcutaneous administration of Iron oxide particles: size dependency," Magnetic Resonance in Medical Sciences, vol. 10, no. 4, pp. 219227, 2011.

[49] C. L. Mallett, C. McFadden, Y. Chen, and P. J. Foster, "Migration of iron-labeled KHYG-1 natural killer cells to subcutaneous tumors in nude mice, as detected by magnetic resonance imaging," Cytotherapy, vol. 14, no. 6, pp. 743-751, 2012.

[50] F. Chen, P. Huang, Y.-J. Zhu, J. Wu, C.-L. Zhang, and D.-X. Cui, "The photoluminescence, drug delivery and imaging properties of multifunctional $\mathrm{Eu}^{3+} / \mathrm{Gd}^{3+}$ dual-doped hydroxyapatite nanorods," Biomaterials, vol. 32, no. 34, pp. 9031-9039, 2011. 
[51] F. Chen, P. Huang, Y.-J. Zhu, J. Wu, and D.-X. Cui, "Multifunctional Eu 3+/Gd 3+ dual-doped calcium phosphate vesiclelike nanospheres for sustained drug release and imaging," Biomaterials, vol. 33, no. 27, pp. 6447-6455, 2012.

[52] R. E. Port, C. Schuster, C. R. Port, and P. Bachert, "Simultaneous sustained release of fludarabine monophosphate and Gd-DTPA from an interstitial liposome depot in rats: Potential for indirect monitoring of drug release by magnetic resonance imaging," Cancer Chemotherapy and Pharmacology, vol. 58, no. 5, pp. 607617, 2006.

[53] V. P. Torchilin, V. S. Trubetskoy, A. M. Milshteyn et al., "Targeted delivery of diagnostic agents by surface-modified liposomes," Journal of Controlled Release, vol. 28, no. 1-3, pp. 45-58, 1994.

[54] E. R. Wisner, K. L. Aho-Sharon, M. J. Bennett, S. G. Penn, C. B. Lebrilla, and M. H. Nantz, "A modular lymphographic magnetic resonance imaging contrast agent: Contrast enhancement with DNA transfection potential," Journal of Medicinal Chemistry, vol. 40, no. 25, pp. 3992-3996, 1997.

[55] M. Levin-Arama, L. Abraham, T. Waner et al., "Subcutaneous compared with intraperitoneal ketamine-xylazine for anesthesia of mice," Journal of the American Association for Laboratory Animal Science, vol. 55, no. 6, pp. 794-800, 2016.

[56] R. E. Borchard, C. D. Barnes, and L. G. Eltherington, Drug Dosage in Laboratory Animals: A Handbook, Telford Press, Caldwell, NJ, USA, 1990.

[57] T. Nevalainen, I. Dontas, A. Forslid et al., "FELASA recommendations for the education and training of persons carrying out animal experiments (category B): report of the federation of european laboratory animal science associations working group on education of persons carrying out animal experiments (category B) accepted by the FELASA board of management," Laboratory Animals, vol. 34, no. 3, pp. 229-235, 2000.

[58] G. Lukas, S. D. Brindle, and P. Greengard, "The route of absorption of intraperitoneally administered compounds," Journal of Pharmacology and Experimental Therapeutics, vol. 178, pp. 562$566,1971$. 


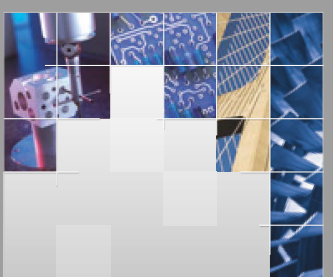

\section{Enfincering}
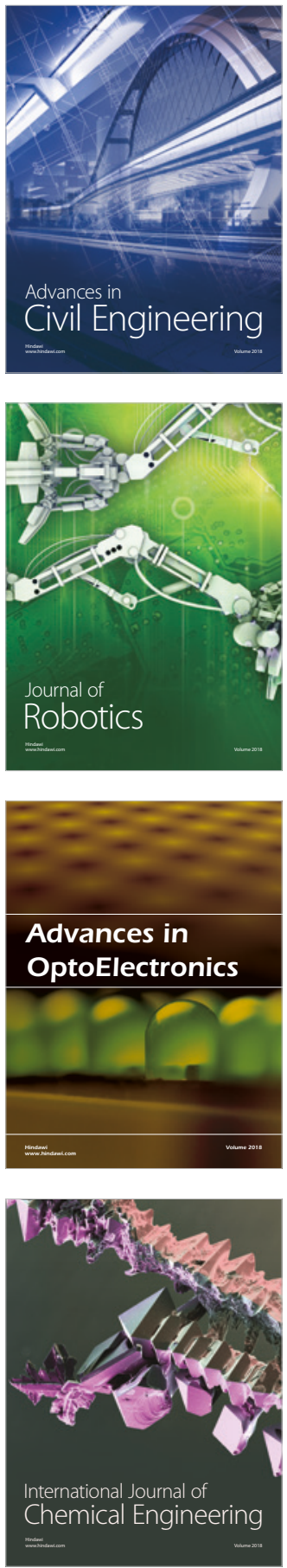

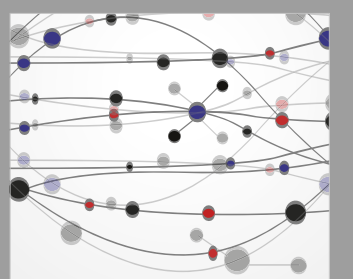

\section{Rotating \\ Machinery}

The Scientific World Journal

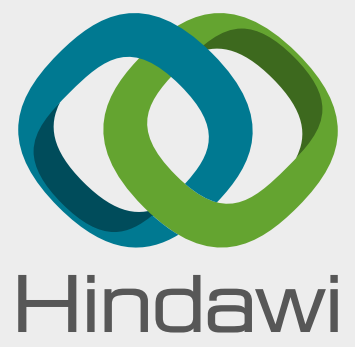

Submit your manuscripts at

www.hindawi.com
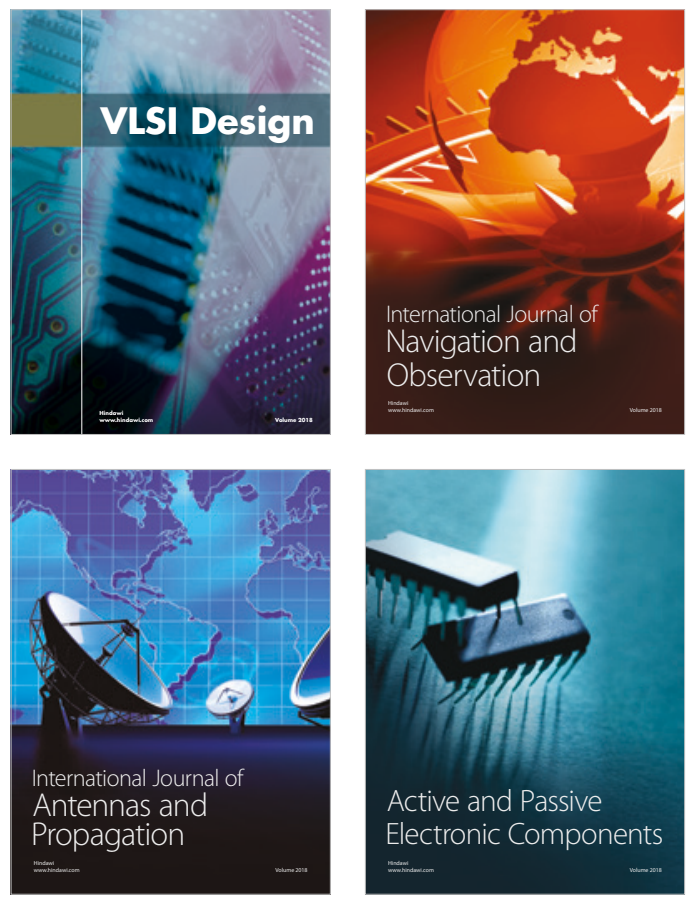
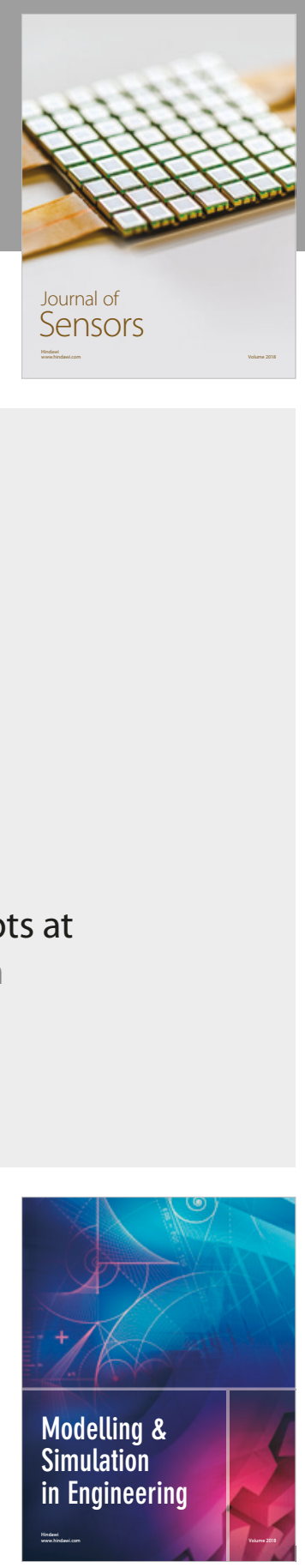

\section{Advances \\ Multimedia}
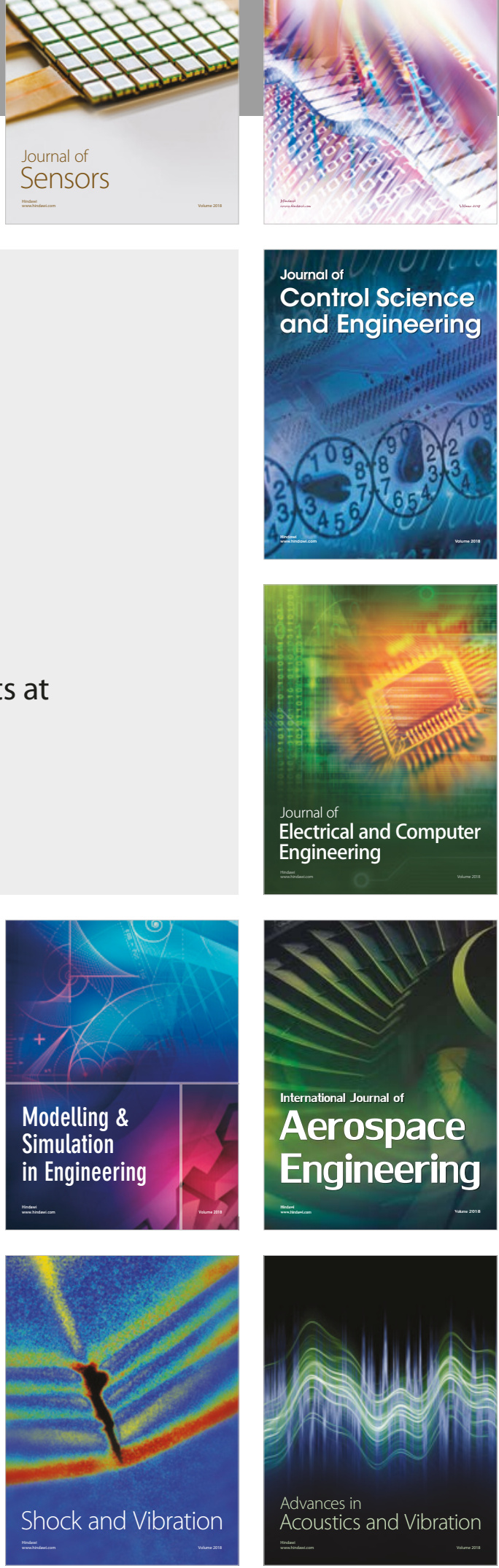